\title{
Vitelliform Macular Dystrophy
}

National Cancer Institute

\section{Source}

National Cancer Institute. Vitelliform Macular Dystrophy. NCI Thesaurus. Code C118788.

A rare genetic disorder characterized by macular degeneration in the retina resulting in progressive loss of central vision with retention of the peripheral vision. It may be of early onset, autosomal dominant inherited and caused by mutations in the BEST 1 gene (BEST disease) or late onset, caused by mutations in the PRPH2 gene. 